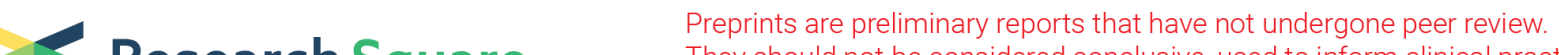 $\begin{array}{ll}\text { Research Square } & \text { They should not be considered conclusive, used to inform clinical practice, } \\ \text { or referenced by the media as validated information. }\end{array}$
}

\section{Postoperative Hypoalbuminemia: Prevalence, Risk Factors, Association with Postoperative Pneumonia in Brain Tumors Patients Undergoing Craniotomy}

\section{Da-wei Zhao}

Third Military Medical University Southwest Hospital

\section{Xu-yang Zhang}

Third Military Medical University Southwest Hospital

\section{Kai-yan Wei}

Third Military Medical University Southwest Hospital

\section{Yi-bin Jiang}

Third Military Medical University Southwest Hospital

\section{Dan Liu}

Third Military Medical University Southwest Hospital

\section{Shui-xian Zhang}

Third Military Medical University Southwest Hospital

\section{Hua Feng}

Third Military Medical University Southwest Hospital

Rong Hu ( $\nabla$ huchrong@tmmu.edu.cn )

Third Military Medical University Southwest Hospital https://orcid.org/0000-0002-6093-9744

\section{Research Article}

Keywords: Brain tumor, Craniotomy, Hypoalbuminemia, Pneumonia, Risk factors

Posted Date: February 15th, 2021

DOI: https://doi.org/10.21203/rs.3.rs-227538/v1

License: (1) (1) This work is licensed under a Creative Commons Attribution 4.0 International License. Read Full License 


\section{Abstract \\ Purpose}

Hypoalbuminemia is associatied with to poor outcome in patients undergoing surgery intervention. The main aim for this study was to investigate the incidence and the risk factors of postoperative hypoalbuminemia and assessed the impact of postoperative hypoalbuminemia on postoperative complications in patients undergoing brain tumor surgery.

\section{Methods}

This retrospective study included 372 consecutive patients who underwent craniotomy for brain tumors from January 2017 to December 2019. The demographic data, pre-and post-operative laboratory tests and postoperative complications were collected. The patients were divided into two groups based on the postoperative serum albumin levels; hypoalbuminemia group $(<35 \mathrm{~g} / \mathrm{L})$ and non-hypoalbuminemia group $(\geq 35 \mathrm{~g} / \mathrm{L}$ ). Univariate and multivariate logistic regression analyses were used to determine risk factors of postoperative hypoalbuminemia and complications.

\section{Results}

Of the total 372 patients underwent craniotomy due to brain tumor, 333 patients ( $89.5 \%$ ) developed hypoalbuminemia after surgery. Hypoalbuminemia was associated with operation time (OR 1.011, P< $0.001)$, preoperative albumin ( $O R=.864, P=0.015)$ and peroperative globulin $(O R 1.192, P=0.004)$. The incidence of postoperative pneumonia in patients with hypoalbuminemia $(41.1 \%)$ significantly higher than that in patients without hypoalbuminemia $(23.1 \%)(P=0.029)$. The independent predictors of postoperative pneumonia were age (OR 1.053, $P<0.001)$, operation time (OR 1.003, $P=0.013)$ and lower postoperative albumin (OR 0.946, $\mathrm{P}=0.018)$.

\section{Conclusions}

Postoperative hypoalbuminemia has a higher incidence with the increase of operation time, and is associated with postoperative pneumonia in patients underwent craniotomy due brain tumor.

\section{Introduction}

Hypoalbuminemia is associated with poor postoperative outcomes and complications in patients underwent surgical intervention, such as cardiac [1, 2], hand [3], colorectal cancer surgery [4] and orthopedic surgery $[5,6]$. In central nervous system, hypoalbuminemia is also associated with increased odds of mortality and a nonroutine hospital discharge $[7,8]$, and is a risk factor for postoperative acute 
kidney injury in patients who underwent craniotomy for tumor [9]. In addition, hypoalbuminemia is independent predictor of extended hospital stay following brain tumor surgery [10].

Postoperative hypoalbuminemia has a considerable incidence in patients underwent craniotomy due to brain tumor in our institution. However, little is known about the association between preoperative clinical parameters and postoperative hypoalbuminemia in patients undergoing brain tumor surgery. Thus, the aim of our study was to investigate the incidence and the risk factors of postoperative albuminemia in patients underwent brain tumor surgery. Furthermore, we assessed the impact of postoperative hypoalbuminemia on postoperative complications after brain tumor surgery.

\section{Subjects And Methods}

\section{Patients}

This retrospective study collected data from 372 consecutive adult patients who underwent craniotomy for brain tumors at the Department of Neurosurgery in the Southwest Hospital of the Third Military Medical University (Army Medical University) from January 2017 to December 2019. All study procedures and protocols involving human participants were in accordance with the ethical standards of the 1964 Helsinki Declaration, and the the Ethics Committee of the Southwest Hospital of Third Military Medical University has approved this study. For retrospective study, formal consent is not required and the requirement for the informed consent of patients was waived prior to the collection of their medical data in this study.

\section{Inclusion and exclusion criteria}

In this study, 372 adult patients with normal preoperative total ( $\geq 60 \mathrm{~g} / \mathrm{L})$ and albumin ( $\geq 35 \mathrm{~g} / \mathrm{L})$, who underwent craniotomy due to intracranial solid tumors, such as gliomas, meningiomas, acoustic neuromas, and metastatic tumors, were included. Patients who underwent craniotomy due to non-solid tumors, such as aneurysm, vascular malformation, intracerebral hemorrhage, hydrocephalus, cranioplasty or skull lesion, had been excluded. Patients younger than 18 years old, who had hypoalbuminemia ( $<35 \mathrm{~g} / \mathrm{L}$ ) before operation were also excluded. Patients with abnormal proteinuria that may lead to protein loss were also excluded. For the purpose of this study, patients with postoperative serum albumin of $<35 \mathrm{~g} / \mathrm{L}$ were defined as hypoalbuminemia group, while those with postoperative serum albumin of $\geq 35 \mathrm{~g} / \mathrm{L}$ were defined as non-hypoalbuminemia group. The postoperative albumin level was checked on the first blood samples taken upon after craniotomy.

\section{Clinical data collection}

For each patients, demographic data [age, sex, body mass index (BMI), histories of smoking and drinking], comorbidities (hypertension, diabetes mellitus, coronary heart disease, viral hepatitis, and history of stroke etc.), as well as pre- and post-operaotive laboratory test were collected. Operation time, intraoperative blood loss, intraoperative total input and intraoperative total urine output composed the 
operative-related medical data. Pathological classification were documented according to Central Nervous System Tumor Classification by the World Health Organization in 2016. The durations of postoperative neurological intensive care unit (NICU) and hospitalization and postoperative complications (pneumonia, epilepsy, incision infection, respiratory failure, renal failure, hydrocephalus, deep vein thrombosis) were also collected.

The pre- and first post-operative (within 6 hours after surgery) laboratory tests included white blood cell count, red blood cell count, hemoglobin, hematocrit, platelet count, fibrinogen (FIB), prothrombin time (PT), prothrombin time-international normalized ratio (PT-INR), activated partial thromboplastin time (APTT), thrombin time (TT), D-dimers, potassium (K), sodium ( $\mathrm{Na}$ ), chloride (Cl), blood glucose, calcium $(\mathrm{Ca})$, phosphorus $(\mathrm{P})$, magnesium $(\mathrm{Mg})$, serum prealbumin, total protein, albumin and albumin/globulin $(A / G)$ ratio.

\section{Statistical analysis}

All statistical analysis was performed using the SPSS software for Windows (version 25.0, IBM SPSS Inc., Chicago, IL) software. Descriptive statistical analysis of the data (e.g., means, medians, frequencies, and percentages) was performed. Normally distributed quantitative variables are presented as the mean \pm standard deviation (SD) and were compared using independent unpaired two-tailed Student's t test. Nonnormally distributed quantitative variables are presented as the median [interquartile range (IQR)] and were compared using independent-sample nonparametric test. Categorical variables were expressed as counts with percentages and compared using the chi-squared test or continuity correction test. Logistics reression analysis was used to investigate the risk factors of postoperative hypoalbuminemia and pneumonia. The variables were analyzed by univariate binary logistic regression analysis. Multicolinearity was assessed using the Pearson correlation coefficient statistic and by checking the Variance Inflation Factor multiple regression model with the same dependent and independent variables. Multivariate logistic regression model was performed on variables with significant differences $(P<0.05)$ determined in the univariate analysis to investigate the relationship between variables and postoperative hypoalbuminemia/pneumonia. All statistical tests of hypothesis performed at the 0.05 level of significance.

\section{Results}

\section{Characteristics of Patients Underwent Craniotomy for Tumor}

A total of 372 patients who underwent craniotomy for solid tumors [161 male (43.3\%), 211 female $(56.7 \%)]$ with a mean age of $48 \pm 13$ years (range 20-86 years) were enrolled. Of these, $333(89.5 \%)$ patients developed hypoalbuminemia, whereas 39 (10.5\%) patients remained normal albumin levels after craniotomy. There were significant statistical differences in BMI $(P<0.05)$ between with and without hypoalbuminemia patients. The patients with hypoalbuminemia had significant greater intraoperative blood loss, intraoperative total input, intraoperative total urine output, operation time and incidence of postoperative pneumonia than those without hypoalbuminemia after craniotomy (all $\mathrm{P}<0.05$ ). There 
were no significant differences in age, sex, previous diseases, lifestyle factors, pathological classification and other complications (all $\mathrm{P}>0.05$ ) between the two groups. The baseline characteristics of the two groups patients are shown in Table 1. There were significant statistical differences in preoperative laboratory tests, including red blood cells, $P T$, albumin, globulin, $A / G$ ratio $(P<0.05)$ between with and without postoperative hypoalbuminemia patients (Table 2).

\section{Predictors Associated with Postoperative Hypoalbuminemia}

In univariate analysis, $\mathrm{BMI}$, intraoperative blood loss, intraoperative total input, intraoperative total urine output, operation time, red blood cells, PT, albumin, globulin and A/G ratio were significantly associated with postoperative hypoalbuminemia (Table 3). The multivariate logistic regression analysis showed that operation time [OR 1.011 (95\% Cl: 1.006-1.016, P < 0.001)], preoperative albumin [OR 0.864 (95\% Cl: $0.768-0.972, \mathrm{P}=0.015)$ ] and peroperative globulin [OR $1.192(95 \% \mathrm{Cl}: 1.058-1.344, \mathrm{P}=0.004)$ ] were independent predictors of postoperative hypoalbuminemia for patients underwent craniotomy due to tumor (Table 4).

\section{Predictors Associated with Postoperative Pneumonia}

The overall incidence rate of postoperative pneumonia was $39.2 \%$ in total of 372 patients underwent craniotomy for brain tumor resection. Table 1 shows that the incidence of postoperative pneumonia in patients with hypoalbuminemia (41.1\%) significantly higher than that in patients without hypoalbuminemia $(23.1 \%)(P=0.029)$. So we compared the baseline characteristics and postoperative laboratory test data of patients with and without postoperative pneumonia (Supplementary table 1), and introduced statistically significant variables into multivariate logistic regression analysis to explore the relevant risk factors of postoperative pneumonia.

The multivariate logistic regression analysis showed that age [OR $1.053(95 \% \mathrm{Cl}: 1.032-1.073, \mathrm{P}<0.001)$, operation time [OR 1.003 (95\% Cl: 1.001-1.005, P = 0.013)] and lower postoperative albumin [OR 0.946 (95\% Cl: 0.903-0.990, $\mathrm{P}=0.018)$ ] were independent predictors of postoperative pneumonia for patients underwent craniotomy due to tumor (Table 5).

\section{Discussion}

In this study, the overall incidence of postoperative hypoalbuminemia was $89.5 \%$ in brain tumor patients after craniotomy surgery. Patients with hypoalbuminemia had a higher intraoperative blood loss, intraoperative total input, intraoperative total urine output, operation time and incidence of postoperative pneumonia compared to the patients without hypoalbuminemia. Logistic regression analysis showed that operation time, preoperative albumin and peroperative globulin were independent risk factors of postoperative hypoalbuminemia in brain tumor postoperative patients. To our knowledge, there are few reports about the incidence and risk factors of postoperative hypoalbuminemia in patients after brain tumor surgery. In addition, the hypoalbuminemic patients showed a higher rate of pneumonia. Increasing 
age, extending operation time and decreasing postoperative albumin were risk factors of postoperative pneumonia.

For the mechanism of hypoalbuminemia, decreased intestinal absorption of protein due to poor oral intake, decreased synthesis of albumin due to hepatic dysfunction, increased catabolism of protein, albuminuria, and extensive vascular leakage of serum protein due to increased capillary permeability have been postulated [11]. In this study, the preoperative albumin of the patients were in the normal levels, and there was no significant differences between with and without postoperative hypoalbuminemia in the related diseases that may affect protein metabolism and loss, such as hepatitis and diabetes. In our institution, we performed a uniform preoperative fasting time for patients who prepared surgery for brain tumor. Therefore, we speculate that the early decrease of albumin may be related to the intraoperative blood loss and hemodilution caused by positive volume infusion [12]. While patients with hypoalbuminemia had lower levels in red blood cells, hemoglobin, hematocrit, platelets, total protein and $A / G$ ratio (data not shown), which also proved that hemodilution may be responsible for dilution hypoalbuminemia. The extended operation time aggravates the occurrence of this process. The results proved that increasing operation time was an independent risk factor for postoperative hypoalbuminemia, which suggest that surgeons should shorten operation time as much as possible to avoid the risk of postoperative hypoalbuminemia and its related poor outcome.

Hypoalbuminemia is associated with poor outcomes in patients undergoing surgical intervention. In central nervous system, preoperative hypoalbuminemia affected the prognoses of patients with glioblastomas $[13,14]$. Hypoalbuminemia is also associated with increased odds of mortality and a nonroutine hospital discharge $[7,8]$, and is a risk factor for postoperative acute kidney injury in patients who underwent craniotomy for tumor [9]. In addition, hypoalbuminemia is independent predictor of extended hospital stay following brain tumor surgery [10].

Postoperative pulmonary complications were significantly associated with poor outcome, including higher reoperation, readmission, mortality, and extended hospital stay in patients that surgical resection of brain tumors $[15,16]$. Previous study identified increasing operative time, increasing age, and increasing estimated blood loss, diabetes, chronic obstructive pulmonary disease, preoperative leukocytosis, American Society of Anesthesiologists classification $\geq 3$, and infratentorial lesions as risk factors for postoperative pneumonia in postcraniotomy patients [15-17]. However, in infratentorial craniotomy with high risk for the development of postoperative pulmonary complications, the predictors for the occurrence of postoperative pulmonary complications were postoperative blood transfusion, lower cranial nerve palsy, prolonged ICU stay and tracheostomy [18]. Our result showed that pneumonia was more frequently incident in patients with hypoalbuminemia than in patients without hypoalbuminemia. Increasing age, extending operative time as risk factors for postoperative pneumonia were consistent with previous studies [15-17]. Postoperative albumin as a novel factors warrant further investigation.

We believe the results from our analysis can provide several benefits to neurosurgeons and neuroanesthesiologists alike. Most evident is the identification of brain tumor patients at risk for 
postoperative hypoalbuminemia and pneumonia, but perhaps more meaningful is the quantification of independent predictors. In addition, the results can provide several suggestions. Surgeons should shorten the operation time as much as possible, while neuroanesthesiologists should refine the management of intraoperative blood volume and respiratory tract.

Nevertheless, our present study also had several limitations. Firstly, this was a single-center retrospective case control study, which has obvious limitations. Secondly, whether preoperative albumin is normal or not as the inclusion criteria may lead to greater heterogeneity of patients. Thirdly, whether albumin supply can change the incidence of pneumonia still needs to be verified by prospective randomized trials.

\section{Conclusions}

The incidence of hypoalbuminemia after craniotomy for brain tumors was high, and the incidence of postoperative pneumonia was higher in patients with hypoalbuminemia. The operation time was common independent risk factors for postoperative hypoalbuminemia and pneumonia. Moreover, our results demonstrate that older age and hypoproteinemia have great potential as predictors of postoperative pneumonia and suggests that clinicians need to provide early management of postoperative hypoproteinemia for the prevention of pneumonia. However, a prospective study is needed to determine whether albumin supply can change the incidence of pneumonia in patients underwent craniotomy due to brain tumor.

\section{Declarations}

Funding: Not applicable

Conflicts of interest: The authors declare that they have no conflict of interest.

Data Availability Statement: The datasets used and analysed to support the findings of current study are available from the corresponding author upon reasonable request.

Code availability: Not applicable

Authors' contributions: RH and FH conceived, organized and supervised the study. DL, SXZ and YBJ collected patient's data. DWZ, XYZ, KYW, YBJ, DL and SXZ conducted the research. DWZ, KYW, XYZ and $\mathrm{RH}$ performed the statistical analysis. $\mathrm{XYZ}, \mathrm{YBJ}, \mathrm{DL}$ and $\mathrm{SXZ}$ prepared the tables. DWZ, $\mathrm{XYZ}$ and $\mathrm{RH}$ prepared and revised the manuscript. All authors approved the final version to be published.

Ethics approval: This study adheres to the principles of the Declaration of Helsinki. The study protocol was approved by the Ethics Committee of the Southwest Hospital of Third Military Medical University, China.

\section{Informed consent}


Informed consent was not sought as a retrospective design was used.

\section{Acknowledgement}

We thank the Department of Epidemiology of the Military Medical Institute at the People's Liberation Army of China for technical assistance in statistical analysis.

\section{References}

1. Berbel-Franco D, Lopez-Delgado JC (2020) The influence of postoperative albumin levels on the outcome of cardiac surgery. J Cardiothorac Surg 15: 78. https://doi.org/10.1186/s13019-020-01133y

2. Putzu A, Esteve F, Torrado H, Farrero E, Rodríguez-Castro D, Carrio ML, Landoni G, Lee EH, Chin JH, Choi DK, Hwang BY, Choo SJ, Song JG, Kim TY, Choi IC (2011) Postoperative hypoalbuminemia is associated with outcome in patients undergoing off-pump coronary artery bypass graft surgery. $J$ Cardiothorac Surg 25: 462-468. https://doi.org/10.1186/s13019-020-01133-y

3. Luchetti TJ, Chung A, Olmscheid N, BohI DD, Hustedt JW (2020) Hypoalbuminemia Is Associated With Increased Postoperative Mortality and Complications in Hand Surgery. Hand 15: 547-555. https://doi.org/10.1177/1558944718820959

4. Hu WH, Eisenstein S, Parry L, Ramamoorthy S (2019) Preoperative malnutrition with mild hypoalbuminemia associated with postoperative mortality and morbidity of colorectal cancer: a propensity score matching study. Nutr J 18: 33. https://doi.org/10.1186/s12937-019-0458-y

5. Shin $\mathrm{KH}$, Han SB (2018) Early postoperative hypoalbuminemia is a risk factor for postoperative acute kidney injury following hip fracture surgery. Injury 49: 1572-1576. https://doi.org/10.1016/j.injury.2018.05.001

6. Vora M, Sing DC, Yi PH, Cheah JW, Li X (2020) Hypoalbuminemia is a risk factor for predicting early postoperative complications after proximal humerus fracture fixation. J Orthop 19: 106-110. https://doi.org/10.1016/j.jor.2019.11.022

7. Borg N, Guilfoyle MR, Greenberg DC, Watts C, Thomson S (2011) Serum albumin and survival in glioblastoma multiforme. J Neurooncol 105: 77-81. https://doi.org/10.1007/s11060-011-0562-0

8. Dasenbrock HH, Liu KX, Chavakula V, Devine CA, Gormley WB, Claus EB, Smith TR, Dunn IF (2017) Body habitus, serum albumin, and the outcomes after craniotomy for tumor: a National Surgical Quality Improvement Program analysis. J Neurosurg 126: 677-689. https://doi.org/10.3171/2016.2.jns152345

9. Kim K, Bang JY, Kim SO, Kim S, Kim JU, Song JG (2018) Association of preoperative hypoalbuminemia with postoperative acute kidney injury in patients undergoing brain tumor surgery: a retrospective study. J Neurosurg 128: 1115-1122. https://doi.org/10.3171/2016.11.jns162237

10. Dasenbrock HH, Liu KX, Devine CA, Chavakula V, Smith TR, Gormley WB, Dunn IF (2015) Length of hospital stay after craniotomy for tumor: a National Surgical Quality Improvement Program analysis. 
Neurosurg Focus 39: E12. https://doi.org/10.3171/2015.10.focus15386

11. Soeters PB, Wolfe RR, Shenkin A (2019) Hypoalbuminemia: Pathogenesis and Clinical Significance. J Parenter Enteral Nutr 43: 181-193. https://doi.org/10.1002/jpen.1451

12. Kreimeier U, Messmer K (2002) Perioperative hemodilution. Transfus Apher Sci 27: 59-72. https://doi.org/10.1016/s1473-0502(02)00027-7

13. Han S, Huang Y, Li Z, Hou H, Wu A (2015) The prognostic role of preoperative serum albumin levels in glioblastoma patients. BMC cancer 15: 108. https://doi.org/10.1186/s12885-015-1125-0

14. Marini A, Dobran M, Aiudi D, Pesaresi A, di Somma LGM, lacoangeli M (2020) Pre-operative hematological markers as predictive factors for overall survival and progression free survival in glioblastomas. Clin Neurol Neurosurg 197: 106-162. https://doi.org/10.1016/j.clineuro.2020.106162

15. Longo M, Agarwal V (2019) Postoperative Pulmonary Complications Following Brain Tumor Resection: A National Database Analysis. World Neurosurg 126: e1147-e1154. https://doi.org/10.1016/j.wneu.2019.03.058

16. Oh T, Safaee M, Sun MZ, Garcia RM, McDermott MW, Parsa AT, Bloch O (2014) Surgical risk factors for post-operative pneumonia following meningioma resection. Clin Neurol Neurosurg 118: 76-79. https://doi.org/10.1016/j.clineuro.2013.12.017

17. Zhang D, Zhuo H, Yang G, Huang H, Li C, Wang X, Zhao S, Moliterno J, Zhang Y (2020) Postoperative pneumonia after craniotomy: incidence, risk factors and prediction with a nomogram. J Hosp Infect 105: 167-175. https://doi.org/10.1016/j.jhin.2020.03.015

18. Hooda B, Chouhan RS, Rath GP, Lamsal R, Bithal PK (2019) Incidence and predictors of postoperative pulmonary complications in patients undergoing craniotomy and excision of posterior fossa tumor. $\mathrm{J}$ Anaesthesiol Clin Pharmacol 35: 254-260. https://doi.org/10.4103/joacp.JOACP_350_17

\section{Tables}

Table 1. Demographic and clinical characteristics between with and without hypoabluminemia patients underwent craniotomy for brain tumor 


\begin{tabular}{|c|c|c|c|}
\hline Variables & $\begin{array}{l}\begin{array}{l}\text { Non- } \\
\text { hypoalbuminemia } \\
N=39(10.5 \%)\end{array}\end{array}$ & $\begin{array}{l}\text { Hypoalbuminemia } \\
\mathrm{N}=333(89.5 \%)\end{array}$ & $\begin{array}{l}P \\
\text { value }\end{array}$ \\
\hline Age (years, mean $\pm S D$ ) & $46 \pm 13$ & $48 \pm 13$ & 0.311 \\
\hline $\operatorname{Sex}[M, n(\%)]$ & $20(51.3)$ & $141(42.3)$ & 0.286 \\
\hline BMI & $24.9 \pm 2.7$ & $23.4 \pm 3.3$ & 0.010 \\
\hline \multicolumn{4}{|l|}{ Medical history [n (\%)] } \\
\hline Hypertension & $5(12.8)$ & $57(17.1)$ & 0.496 \\
\hline Diabetes & $2(5.1)$ & $12(3.6)$ & 0.636 \\
\hline Coronary heart disease & $2(5.1)$ & $5(1.5)$ & 0.115 \\
\hline Viral hepatitis & $1(2.6)$ & $11(3.3)$ & 0.805 \\
\hline History of stroke & $1(2.6)$ & $3(0.9)$ & 0.341 \\
\hline \multicolumn{4}{|l|}{ Lifestyle factors [n (\%)] } \\
\hline Smoking & $12(30.8)$ & $82(24.6)$ & 0.403 \\
\hline Drinking & $4(10.3)$ & $36(10.8)$ & 0.916 \\
\hline \multicolumn{4}{|l|}{ Pathological classification [n (\%)] } \\
\hline Meningiomas & 13 (33.3) & $131(39.3)$ & 0.288 \\
\hline Gliomas & $9(23.1)$ & $111(33.3)$ & \\
\hline Acoustic neuromas & $3(7.7)$ & $21(6.3)$ & \\
\hline Metastatic tumors & $3(7.7)$ & $15(4.5)$ & \\
\hline Others & $11(28.2)$ & $55(16.5)$ & \\
\hline Intraoperative blood loss [(mL, Median (IQR)] & $200(200-300)$ & $300(300-500)$ & $<0.001$ \\
\hline Intraoperative total input [(mL, Median (IQR)] & $2200(1813-2688)$ & $2850(2300-3500)$ & $<0.001$ \\
\hline $\begin{array}{l}\text { Intraoperative total urine output }[(\mathrm{mL}, \text { Median } \\
(\mathrm{IQR})]\end{array}$ & $1775(1400-2500)$ & $2600(2015-3350)$ & $<0.001$ \\
\hline Operation time [(h, Median (IQR)] & $3.8(2.7-5.0)$ & $5.0(4.0-6.0)$ & $<0.001$ \\
\hline NICU time [(days, Median (IQR)] & $4(3-5)$ & $4(3-5)$ & 0.068 \\
\hline Hospitalization time [(days, Median (IQR)] & $21(17-28)$ & $21(18-28)$ & 0.524 \\
\hline \multicolumn{4}{|l|}{ Postoperative complications [n (\%)] } \\
\hline Pneumonia & $9(23.1)$ & $137(41.1)$ & 0.029 \\
\hline
\end{tabular}




\begin{tabular}{|llll|}
\hline Epilepsy & $3(7.7)$ & $10(3.0)$ & 0.131 \\
\hline Incision infection & - & $3(0.9)$ & 0.552 \\
\hline Respiratory failure & - & $11(3.3)$ & 0.249 \\
\hline Renal failure & - & $3(0.9)$ & 0.552 \\
\hline Hydrocephalus & - & $3(0.9)$ & 0.552 \\
\hline Deep vein thrombosis & - & $2(0.6)$ & 0.627 \\
\hline
\end{tabular}

BMI, Body Mass Index; NICU, Neurological Intensive Care Unit; SD, standard deviation;IQR, interquartile range

Table 2. Comparison of preoperative laboratory findings between with and without hypoabluminemia patients underwent craniotomy for brain tumor (mean \pm SD) 


\begin{tabular}{|c|c|c|c|}
\hline Variables & $\begin{array}{l}\text { Non-hypoalbuminemia } \\
\mathrm{N}=39(10.5 \%)\end{array}$ & $\begin{array}{l}\text { Hypoalbuminemia } \\
\mathrm{N}=333(89.5 \%)\end{array}$ & $P$ value \\
\hline White blood cells $\left(10^{9} / \mathrm{L}\right)$ & $6.13 \pm 1.46$ & $6.65 \pm 2.45$ & 0.198 \\
\hline Red blood cells, $\left(10^{12} / \mathrm{L}\right)$ & $4.79 \pm 0.61$ & $4.62 \pm 0.48$ & 0.046 \\
\hline Hemoglobin (g/L) & $141 \pm 17$ & $137 \pm 15$ & 0.146 \\
\hline Hematocrit (\%) & $42.9 \pm 4.8$ & $41.9 \pm 4.2$ & 0.147 \\
\hline Platelets $\left(10^{9} / \mathrm{L}\right)$ & $216 \pm 61$ & $215 \pm 61$ & 0.933 \\
\hline $\mathrm{FIB}(\mathrm{g} / \mathrm{L})$ & $2.39 \pm 0.48$ & $2.57 \pm 0.84$ & 0.204 \\
\hline PT (sec) & $10.75 \pm 0.52$ & $11.00 \pm 0.74$ & 0.041 \\
\hline PT-INR & $0.93 \pm 0.05$ & $0.95 \pm 0.07$ & 0.052 \\
\hline APTT (sec) & $27.33 \pm 2.82$ & $27.72 \pm 3.73$ & 0.523 \\
\hline TT (sec) & $17.07 \pm 1.09$ & $17.04 \pm 1.17$ & 0.896 \\
\hline D-dimer (mg/L) & $0.23 \pm 0.27$ & $0.40 \pm 1.14$ & 0.352 \\
\hline $\mathrm{K}(\mathrm{mmol} / \mathrm{L})$ & $3.97 \pm 0.39$ & $4.03 \pm 0.32$ & 0.252 \\
\hline $\mathrm{Na}(\mathrm{mmol} / \mathrm{L})$ & $139.9 \pm 2.4$ & $140.0 \pm 2.5$ & 0.569 \\
\hline $\mathrm{Cl}(\mathrm{mmol} / \mathrm{L})$ & $105.5 \pm 2.4$ & $104.8 \pm 2.9$ & 0.160 \\
\hline Creatinine $(\mu \mathrm{mol} / \mathrm{L})$ & $62.4 \pm 13.9$ & $64.2 \pm 13.8$ & 0.425 \\
\hline Glucose (mmol/L) & $5.74 \pm 1.29$ & $5.71 \pm 1.51$ & 0.905 \\
\hline $\mathrm{Ca}(\mathrm{mmol} / \mathrm{L})$ & $2.40 \pm 0.12$ & $2.39 \pm 0.19$ & 0.763 \\
\hline $\mathrm{P}(\mathrm{mmol} / \mathrm{L})$ & $1.15 \pm 0.17$ & $1.20 \pm 0.22$ & 0.150 \\
\hline $\mathrm{Mg}(\mathrm{mmol} / \mathrm{L})$ & $0.88 \pm 0.05$ & $0.89 \pm 0.08$ & 0.656 \\
\hline Prealbumin $(\mathrm{g} / \mathrm{L})$ & $0.26 \pm 0.05$ & $0.25 \pm 0.06$ & 0.221 \\
\hline Total protein $(\mathrm{g} / \mathrm{L})$ & $72.3 \pm 5.3$ & $72.6 \pm 5.6$ & 0.743 \\
\hline Albumin $(\mathrm{g} / \mathrm{L})$ & $44.3 \pm 3.5$ & $43.1 \pm 3.6$ & 0.036 \\
\hline Globulin (g/L) & $27.9 \pm 4.1$ & $29.5 \pm 4.0$ & 0.019 \\
\hline Albumin/globulin ratio & $1.62 \pm 0.27$ & $1.48 \pm 0.22$ & $<0.001$ \\
\hline
\end{tabular}

FIB, fibrinogen; PT, prothrombin time; PT-INR, prothrombin time-internatioinal normalized ratio; APTT, activated partial thromboplasti time; TT, thrombin time; $\mathrm{K}$, potassium; $\mathrm{Na}$, sodium; $\mathrm{Cl}$, chloride; $\mathrm{Ca}$, calcium; P, phosphorus; Mg, magnesium. 
Table 3. Univariate logistic regression analysis of risk factors influencing postoperative hypoalbuminemia in the patients underwent craniotomy for brain tumor

\begin{tabular}{|llllll|}
\hline Variables & B & SE & Wald & P value & Odds ratio (95\% Cl) \\
\hline BMI & -0.141 & 0.056 & 6.354 & 0.012 & $0.869(0.779-0.969)$ \\
\hline Intraoperative blood loss & 0.002 & 0.001 & 4.295 & 0.038 & $1.002(1.000-1.004)$ \\
\hline Intraoperative total input & 0.001 & 0.000 & 15.191 & $<0.001$ & $1.001(1.000-1.001)$ \\
\hline Intraoperative total urine output & 0.001 & 0.000 & 17.178 & $<0.001$ & $0.001(1.001-1.002)$ \\
\hline Operation time & 0.008 & 0.002 & 16.073 & 0.000 & $1.009(1.004-1.013)$ \\
\hline Red blood cells & -0.640 & 0.324 & 3.915 & 0.048 & $0.527(0.280-0.994)$ \\
\hline PT & 0.613 & 0.292 & 4.424 & 0.035 & $1.846(1.043-3.269)$ \\
\hline Albumin & -0.098 & 0.047 & 4.355 & 0.037 & $0.906(0.826-0.994)$ \\
\hline Globulin & 0.109 & 0.047 & 5.470 & 0.019 & $1.115(1.018-1.221)$ \\
\hline Albumin/globulin ratio & -2.400 & 0.700 & 11.761 & 0.001 & $0.091(0.023-0.358)$ \\
\hline
\end{tabular}

BMI, Body Mass Index; B, regression coefficient; SE., standard errors of regression coefficient; PT, prothrombin time.

Table 4. Multivariate logistic regression analysis of risk factors influencing postoperative hypoalbuminemia in the patients underwent craniotomy for brain tumor

\begin{tabular}{|llllll|}
\hline Variables & B & SE & Wald & P value & Odds ratio $(95 \%$ Cl) \\
\hline Operation time & 0.001 & 0.003 & 18.118 & $<0.001$ & $1.011(1.006-1.016)$ \\
\hline Peroperative albumin & -0.146 & 0.060 & 5.922 & 0.015 & $0.864(0.768-0.972)$ \\
\hline Peroperative globulin & 0.176 & 0.061 & 8.294 & 0.004 & $1.192(1.058-1.344)$ \\
\hline
\end{tabular}

B, regression coefficient; SE., standard errors of regression coefficient.

Table 5. Multivariate logistic regression analysis of risk factors influencing postoperative pneumonia in the patients underwent craniotomy for brain tumor

\begin{tabular}{|llllll|}
\hline Variables & B & SE & Wald & P value & Odds ratio (95\% Cl) \\
\hline Age & 0.051 & 0.010 & 27.100 & $<0.001$ & $1.053(1.032-1.073)$ \\
\hline Operation time & 0.003 & 0.001 & 6.145 & 0.013 & $1.003(1.001-1.005)$ \\
\hline Postoperative albumin & -0.056 & 0.024 & 5.631 & 0.018 & $0.946(0.903-0.990)$ \\
\hline
\end{tabular}


B, regression coefficient; SE., standard errors of regression coefficient

\section{Supplementary Files}

This is a list of supplementary files associated with this preprint. Click to download.

- Supplementarytable.docx 